\title{
Assessing the transcriptional regulation of L-cysteine desulfhydrase 1 in Arabidopsis thaliana
}

\author{
Ana M. Laureano-Marín, Irene García, Luis C. Romero and Cecilia Gotor* \\ Instituto de Bioquímica Vegetal y Fotosíntesis, Consejo Superior de Investigaciones Científicas and Universidad de Sevilla, Sevilla, Spain
}

\section{Edited by:}

Stanislav Kopriva, University of

Cologne, Germany

Reviewed by:

Anna Wawrzynska, Institute of

Biochemistry and Biophysics Polish

Academy of Sciences, Poland

Min-jie Cao, Chinese Academy of

Sciences, China

${ }^{*}$ Correspondence:

Cecilia Gotor, Instituto de

Bioquímica Vegetal y Fotosíntesis,

Avenida Américo Vespucio, 49,

41092 Sevilla, Spain

e-mail: gotor@ibvf.csic.es
Hydrogen sulfide is an important signaling molecule that functions as a physiological gasotransmitter of comparable importance to $\mathrm{NO}$ and $\mathrm{CO}$ in mammalian systems. In plants, numerous studies have shown that sulfide increases tolerance/resistance to stress conditions and regulates essential processes. The endogenous production of hydrogen sulfide in the cytosol of Arabidopsis thaliana occurs by the enzymatic desulfuration of L-cysteine, which is catalyzed by the L-cysteine desulfhydrase enzyme DES1. To define the functional role of DES1 and the role that the sulfide molecule may play in the regulation of physiological processes in plants, we studied the localization of the expression of this gene at the tissue level. Transcriptional data reveal that DES1 is expressed at all developmental stages and is more abundant at the seedling stage and in mature plants. At the tissue level, we analyzed the expression of a GFP reporter gene fused to promoter of DES1. The GFP fluorescent signal was detected in the cytosol of both epidermal and mesophyll cells, including the guard cells. GFP fluorescence was highly abundant around the hydathode pores and inside the trichomes. In mature plants, fluorescence was detected in floral tissues; a strong GFP signal was detected in sepals, petals, and pistils. When siliques were examined, the highest GFP fluorescence was observed at the bases of the siliques and the seeds. The location of GFP expression, together with the identification of regulatory elements within the DES1 promoter, suggests that DES1 is hormonally regulated. An increase in DES1 expression in response to ABA was recently demonstrated; in the present work, we observe that in vitro auxin treatment significantly repressed the expression of DES1.

Keywords: abscission zone, auxin, DES1 promoter, hydathode, floral tissues, promoter-GFP construct

\section{INTRODUCTION}

Hydrogen sulfide, a known toxic molecule, is considered to be an important signaling molecule. In animal systems, hydrogen sulfide functions as physiological gasotransmitter; this molecule is recognized to be of equal importance to $\mathrm{NO}$ and $\mathrm{CO}$ and has been the subject of many reviews (Gadalla and Snyder, 2010; Kimura, 2011; Wang, 2012). $\mathrm{H}_{2} \mathrm{~S}$ is mostly catalyzed via the enzymatic reactions of cystathionine $\beta$-synthase (CBS) and cystathionine $\gamma$ lyase (CSE) (Wang, 2012) in mammals. Both enzymes are known for their participation in the transsulfuration pathway, which is critical for the synthesis of cysteine from methionine. Both CBS and CSE use pyridoxal 5'-phosphate as a cofactor and are exclusively located in the cytosol (Gadalla and Snyder, 2010; Wang, 2012).

In recent years, hydrogen sulfide has been also shown to be a signaling molecule in plants similar to $\mathrm{NO}$ and $\mathrm{H}_{2} \mathrm{O}_{2}$. Numerous studies have demonstrated the role of sulfide in protection against numerous stress conditions. Additional studies have demonstrated that this molecule is involved in regulating essential processes such as photosynthesis, stomatal movement, senescence, and autophagy. Consequently, several reviews in plant systems have been recently released (Garcia-Mata and Lamattina,
2013; Lisjak et al., 2013; Calderwood and Kopriva, 2014; Gotor et al., 2014; Hancock and Whiteman, 2014).

Hydrogen sulfide is biosynthesized in plant chloroplasts during the photosynthetic sulfate assimilatory process by the sulfite reductase that reduces sulfite to sulfide. Due to the high toxicity of hydrogen sulfide, it is rapidly incorporated into carbon skeletons to form cysteine by the O-acetylserine(thiol)lyase (OASTL) enzymes. OASTL enzymes are found in the cytosol, plastids and mitochondria and are encoded in Arabidopsis thaliana by the $O A S-A 1, O A S-B$, and $O A S-C$ genes, respectively (Takahashi et al., 2011; Romero et al., 2014). In mitochondria, $\mathrm{H}_{2} \mathrm{~S}$ is also produced during the detoxification of cyanide by $\beta$-cyanoalanine synthase; this enzyme catalyzes the conversion of cysteine and cyanide to hydrogen sulfide and $\beta$-cyanoalanine. Like cyanide, sulfide is a potent inhibitor of mitochondrial cytochrome $c$ oxidase. Sulfide in the mitochondria must be detoxified by OAS-C to produce cysteine, thus generating a cyclic pathway for cyanide/sulfide detoxification (Garcia et al., 2010; Alvarez et al., 2012b).

$\mathrm{H}_{2} \mathrm{~S}$ is also produced in plants by cysteine-degrading enzymes, such as D- and L-cysteine desulfhydrases; these enzymes also produce pyruvate and ammonium (Riemenschneider et al., 2005; Alvarez et al., 2010). We have recently shown that the 


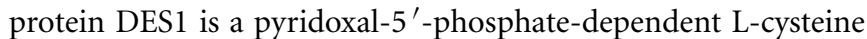
desulfhydrase located in the cytosol of Arabidopsis (Alvarez et al., 2010). Therefore, the $\mathrm{H}_{2} \mathrm{~S}$ levels in the cytosol are determined via the coordinated enzymatic activities of OAS-A1 and DES1 (Gotor et al., 2014; Romero et al., 2014).

Hydrogen sulfide is weakly acidic and dissociates in aqueous solutions into $\mathrm{H}^{+}$and $\mathrm{HS}^{-}$. In this ionized form, hydrogen sulfide cannot permeate membranes (Kabil and Banerjee, 2010). In the basic $\mathrm{pH}$ of the chloroplast stroma under illumination, and in the mitochondrial stroma in metabolically active cells, sulfide is predominantly found in the charged $\mathrm{HS}^{-}$form. Therefore, hydrogen sulfide is unable to cross out the chloroplast and mitochondrial membranes. Accordingly, DES1 is the responsible for the production of sulfide in the plant cytosol (Romero et al., 2013), with an estimated steady-state concentration of $50 \mu \mathrm{M}$ (Krueger et al., 2009).

Recent studies have concluded that DES1 modulates the generation of sulfide for signaling in important plant processes, such as the progression of autophagy and the stomatal movement. Irrespective of nutrient conditions, it was demonstrated that sulfide exerts a general effect on autophagy in plants through negative regulation of this process (Alvarez et al., 2012a; Gotor et al., 2013). It has been recently demonstrated that sulfide generated by DES1 acts upstream of nitric oxide in the ABA signaling network in stomatal guard cells (Scuffi et al., 2014).

To gain insight into the regulation of DES1, we analyzed the tissue and cellular localization of DES1 using a DES1 promoterGFP construct. We found maximum levels of gene expression in the seedling and mature stages of plant development. We were able to further localize the GFP signal to vegetative and reproductive tissues in correlation with the hormonal regulation of DES1.

\section{MATERIALS AND METHODS \\ PLANT MATERIAL, GROWTH CONDITIONS AND TREATMENTS}

Arabidopsis thaliana wild type ecotype Col-0 and the transgenic PromDES1-GFP line were used in this work. Plants were grown in soil for 6 weeks with a photoperiod of $16 \mathrm{~h}$ of white light $(120 \mu \mathrm{E}$ $\mathrm{m}^{-2} \mathrm{~s}^{-1}$ ) at $20^{\circ} \mathrm{C}$ and $8 \mathrm{~h}$ of dark at $18^{\circ} \mathrm{C}$. Alternatively, surface sterilized seeds were germinated and grown in agar-supplemented Murashige and Skoog (MS) medium for 1-2 weeks. For the auxin treatments, wild type Col-0 seeds were germinated and grown for 7 days on MS plates in the presence of 0.1 or $1 \mu \mathrm{M}$ of indoleacetic acid (IAA).

\section{DNA CLONING AND PLASMID CONSTRUCTION}

To clone the DES1 promoter, a $3 \mathrm{~kb}$ of the genomic sequence upstream from the DES1 gene start codon was amplified using specific primers. Total DNA was isolated from young Arabidopsis leaves using the Qiagen DNeasy Plant Minikit. The $3 \mathrm{~kb}$ sequence containing the DES1 promoter was amplified by PCR using the primers proDES1-F: CACCCATTTTATTTTACACCACG and proDES1-R: GTGGTTTGTCTTTGGAAAACT and the Invitrogen proofreading Platinum Pfx DNA polymerase. PCR conditions were as follows: a denaturation cycle of $2 \mathrm{~min}$ at $94^{\circ} \mathrm{C}$, followed by 35 amplification cycles of $15 \mathrm{~s}$ at $94^{\circ} \mathrm{C}, 30 \mathrm{~s}$ at $55^{\circ} \mathrm{C}$, and $1 \mathrm{~min}$ at $68^{\circ} \mathrm{C}$. The amplified region was then ligated into the Invitrogen $\mathrm{pENTR/D-TOPO}$ vector using the Invitrogen Directional TOPO Cloning Kit following the manufacturer's instructions. Positive clones were identified by PCR and chosen for plasmid DNA isolation. Using Invitrogen Gateway ${ }^{\circledR}$ technology, the DES1 promoter was then cloned into the pMDC110 vector (Curtis and Grossniklaus, 2003), a plant expression vector for the construction of promoter-reporter GFP vectors. The final construct for used for plant transformation was identified by colony PCR and plasmid PCR. The construction was named PromDES1-GFP.

\section{TRANSFORMATION OF ARABIDOPSIS}

For plant transformation, the construct PromDES1-GFP was transformed into an Agrobacterium tumefaciens strain and then introduced into $A$. thaliana plants by dipping the developing floral tissues into a solution containing the A. tumefaciens strain, 5\% sucrose, and 0.005\% (v/v) of the surfactant Silwet L-77 (Clough and Bent, 1998). Transgenic plants were recovered by selecting seeds on solid MS medium containing $50 \mathrm{mg} / \mathrm{l}$ of hygromycin.

\section{REAL-TIME RT-PCR}

Quantitative real-time RT-PCR was used to analyze the expression of DES1 and OAS-A1 genes. Total RNA was extracted from different tissues of Arabidopsis plants or the aerial parts of Arabidopsis seedlings using the Qiagen RNeasy Plant Mini Kit. RNA was reverse transcribed using an oligo(dT) primer and the Invitrogen SuperScript First-Strand Synthesis System for RT-PCR following manufacturer's instructions. Gene-specific primers for each gene were designed using the Invitrogen Vector NTI Advance 10 software. Primer sequences were as follows: qDES1-F, 5' ${ }^{\prime}$ TCGAGTCAGTCAGATATGAAGCT-3' and qDES1-R, 5'-TGTAACCTTGGTACCAACATCTCT-3' for the DES1 gene; qOASA-F, 5'-CACGAGCGATTTTCTCCATT-3' and qOASA-R, $5^{\prime}$-CAATTCTCGAGGCCATGATT- $3^{\prime}$ for the OAS-A1 gene; qUBQ-F, 5'-GGCCTTGTATAATCCCTGATGAATAAG-3' and qUBQ-R, 5'-AAAGAGATAACAGGAACGGAAACATAGT$3^{\prime}$ for the constitutive UBQ10 gene. The PCR efficiency of all primer pairs was determined to be close to $100 \%$. Real-time PCR was performed using the Bio-Rad iQ SYBR Green Supermix. Signals were detected on a Bio-Rad iCYCLER according to the manufacturer's instructions. The cycling profile consisted of $95^{\circ} \mathrm{C}$ for $10 \mathrm{~min}$ followed by 45 cycles of $95^{\circ} \mathrm{C}$ for $15 \mathrm{~s}$ and $60^{\circ} \mathrm{C}$ for $1 \mathrm{~min}$. A melting curve from $60^{\circ} \mathrm{C}$ to $90^{\circ} \mathrm{C}$ was run following the PCR cycling. The expression levels of the genes of interest were normalized to that of the constitutive UBQ10 gene by subtracting the cycle threshold (CT) value of $U B Q 10$ from the CT value of the gene $(\triangle \mathrm{CT})$. The results shown are means $\pm \mathrm{SD}$ of at least three independent RNA samples.

\section{GFP LOCALIZATION BY CONFOCAL MICROSCOPY}

Tissues from Arabidopsis at different developmental stages were visualized using a Leica TCS SP2 spectral confocal microscope. Samples were excited using the $488 \mathrm{~nm}$ line of an argon ion laser; emission was detected between 510 and $580 \mathrm{~nm}$ for GFP imaging (pseudocolored green) and between 620 and $680 \mathrm{~nm}$ for chloroplast autofluorescence (pseudocolored red). The microscopy images were processed using the Leica Confocal Software. 


\section{RESULTS}

\section{ISOLATION OF THE DES1 PROMOTER REGION AND PRODUCTION OF} PROMOTER-REPORTER TRANSGENIC PLANTS

Recent work has suggested that DES1 modulates the generation of sulfide in the cytosol for signaling purposes (Gotor et al., 2013; Romero et al., 2013). Mutations in DES1 result in premature leaf senescence in mature plants, which can be observed at transcriptional and cellular levels; and at the seedling stage, an increased tolerance to abiotic stress is observed (Alvarez et al., 2010, 2012a). To determine the role of DES1 in plant growth and development, we examined the spatial and temporal regulation of DES1 gene expression. For this purpose, promoter-GFP transgenic plants were constructed using a $3002 \mathrm{bp}$ fragment isolated from the DES1 promoter region. This fragment comprises the genomic region upstream from the DES1 gene and its first intron. The intron was included based on a previous report demonstrating that the first intronic region of the OASA1 gene, other member of the OASTL family, includes essential elements for tissue-specific expression (Gutierrez-Alcala et al., 2005). Thus, the DES1 promoter consists of $2836 \mathrm{bp}$ from the intergenic region between DES1 (At5g28030) and the upstream gene At5g28040, 14 bp of the first exon containing the 5'-UTR region, $118 \mathrm{bp}$ of the first intron and $34 \mathrm{bp}$ of the second exon that contains the remainder of the $5^{\prime}$-UTR region, immediately upstream of the translation initiation site (Supplemental
Figure 1; www.arabidopsis.org). The promoter sequence was analyzed for cis-acting regulatory elements using available web tools (AthaMapMan; AGRIS; PLACE). Several binding site motifs were detected, including ABA- and Auxin-related elements and leaf development and senescence-regulatory elements (Table 1).

The DES1 promoter was fused to the GFP gene. The plant transformation construct was named PromDES1-GFP. Six transgenic $A$. thaliana plants were obtained; and homozygous lines were analyzed by laser confocal microscopy for in vivo GFP detection. One T4 line was selected for further studies.

\section{DEVELOPMENTAL DES1 EXPRESSION PROFILES IN ARABIDOPSIS WILD TYPE PLANTS}

To investigate the transcriptional regulation of the DES1 gene, we first examined its expression profile during the development and in different tissues of wild type Arabidopsis plants, using real-time RT-PCR analysis. Tissues were harvested either from seedlings grown on MS plates without sucrose or from plants grown in soil at different growth stages up to maturity (Boyes et al., 2001). The highest DES1 expression levels were detected in leaf tissues at the beginning and end of plant development; this corresponded to 14-day-old seedlings (growth stage 1.04) and to 35-day-old plants (growth stage 8.0). Flowering was completed at growth stage 8.0, at least under our experimental conditions (Figure 1). The lowest $D E S 1$ expression level found in leaves was observed at growth

Table 1 | List of various cis-regulatory elements and their positions in the DES1 promoter.

\begin{tabular}{|c|c|c|c|c|c|}
\hline & Function & cis element & Sequence & Position & TF family \\
\hline 1 & Defense against insect herbivory & AtMYC2 BS in RD22 & CACATG & (1578-1583) & BHLH \\
\hline 2 & Development & Bellringer/Replumless /Pennywise & AAATTAAA & $(2593-2600)$ & Homeobox \\
\hline 3 & Development & Bellringer/Replumless /Pennywise & AAATTAGT & $(1368-1375)$ & Homeobox \\
\hline 4 & Development & Bellringer/Replumless /Pennywise & ACTAATTT & $(293-300)$ & Homeobox \\
\hline 5 & Response to hiperosmolarity & ATB2/AtbZIP53/AtbZIP44/GBF5 BS in ProDH & ACTCAT & $(2537-2542)$ & bZIP \\
\hline 6 & Auxins response & ARFI binding site motif & TGTCTC & $\begin{array}{c}(177-182) \\
(1820-1825)\end{array}$ & ARF \\
\hline 7 & ABA response & DPBF1\&2 binding site motif & ACACTAG & (897-904) & bZIP \\
\hline 8 & & MYB binding site promoter & AACCAAAC & $(2359-2366)$ & MYB \\
\hline 9 & Defense and stress & MYB4 binding site motif & AACAAAC & $\begin{array}{c}(825-831) \\
(775-781) \\
(2446-2452)\end{array}$ & MYB4 \\
\hline 10 & Leaf maturation and senescence & RAVI-A binding site motif & CAACA & $\begin{array}{c}(1-5) \\
(2138-2142) \\
(1508-1512) \\
(583-587) \\
(360-367)\end{array}$ & ABI3VPI \\
\hline 11 & Development & LFY consensus binding site motif & CCAATG & $(1413-1418)$ & LFY \\
\hline 12 & & Boxll promoter motif & GGTTAA & $(2810-2815)$ & \\
\hline 13 & Drought response & DRE-like promoter motif & TACCGACCA & $(533-541)$ & \\
\hline 14 & Light response & GATA promoter motif [LRE] & TGATAG & $(2957-2962)$ & \\
\hline 15 & Light response & GATA promoter motif [LRE] & AGATAA & $\begin{array}{r}(287-292) \\
(96-101)\end{array}$ & \\
\hline 16 & Light response & GATA promoter motif [LRE] & TGATAA & $(2308-2313)$ & \\
\hline 17 & & Hexamer promoter motif & CCGTCG & $(649-654)$ & \\
\hline 18 & & T-box promoter motif & ACTTIG & $(1529-1534)$ & \\
\hline
\end{tabular}

The immediate upstream nucleotide of the start codon is designated as position 1 as shown in the Supplemental Figure 1. 


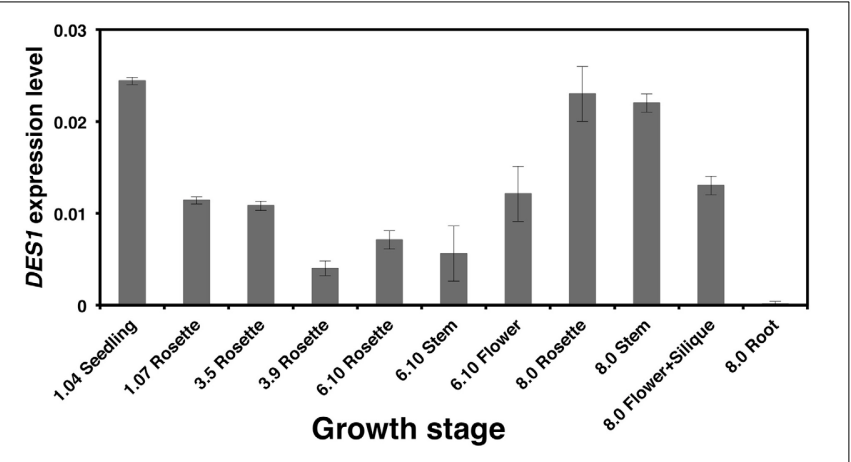

FIGURE 1 | Relative expression levels of the DES1 gene in different tissues at different growth stages. Wild type Arabidopsis plants were grown in soil under the growth conditions described in Materials and Methods. At the indicated growth stages, the tissues were collected for real-time RT-PCR analysis. For the growth stage 1.04, seeds were grown on solid MS medium without sucrose in Petri dishes. The DES1 transcript levels were normalized to an internal control, the constitutively expressed UBQ10 gene. Data shown are mean values \pm SD from three independent analyses.

stage 3.9, which corresponded to plants where rosette growth was complete but before flower buds were visible. Curiously, the DES1 expression levels in reproductive tissues (flowers and siliques) were significantly greater compared to rosette leaves in plants at vegetative growth stages.

\section{GFP EXPRESSION DRIVEN BY THE DES1 PROMOTER IN VEGETATIVE TISSUES}

The tissue-specific expression of the DES1 gene was examined further using the promoter-GFP approach. GFP was visualized in PromDES1-GFP plants using confocal microscopy. GFP expression largely correlated with DES1 expression profiles in wild type plants at the whole tissue level. At the seedling stage, we detected GFP fluorescence in the whole leaf; fluorescence was observed initially at 7 days after sowing (Figure 2). A closer examination of the abaxial side of the leaf revealed some specific sites with high GFP accumulation. High expression at the very tip of the leaf would correspond with hydathode pores (Figures 2A-D). In epidermal cells, we localized the GFP signal to the thin layer of cytoplasm underneath the cell wall; this included the guard cells of the stomata (Figures 2E,F). The localization of GFP to the nucleus that we observed was likely due to the relatively small size GFP, which can translocate to the nucleus on its own through nuclear pores (Seibel et al., 2007).

GFP localization was also observed throughout the adaxial side of the leaf in 7-day-old seedlings. Obscure zones with no fluorescence corresponded to trichomes growing upwards in the vertical plane (Figures 2G,H). A strong GFP signal was observed inside the trichomes and in the trichome basement cells; GFP was localized to the cytoplasmic strands and clearly detectable (Figure 2I). GFP also appeared in the base of the petiole (Figure 2J). In root tissues, GFP was only observable in the hypocotyl-root transition zone (Figure 2K).

At the 1.04 growth stage (14-day-old seedlings), the GFP signal increased and was distributed throughout the leaf (Figure 3 ).
Accordingly, the maximum GFP localization was associated with the hydathode pores; the larger the leaf, the greater number of hydathodes contained (Figures 3A-C). A closer look at the mesophyll cell layer (Figures 3D,E) and leaf vascular tissues (Figure 3F) also revealed GFP expression. At this growth stage, GFP was detectable in root tissues; fluorescence was mostly observed in the meristematic zone and vascular tissues (Figures 3G-I).

\section{GFP EXPRESSION DRIVEN BY THE DES1 PROMOTER IN REPRODUCTIVE TISSUES}

At the mature stage, the promoter-GFP approach also confirmed previous data concerning DES1 expression at the organ level. A significant level of GFP fluorescence was observed in floral tissues (Figure 4). In open flowers, a strong GFP signal was detected in the upper pistil and at the base of the pistil; only a very weak signal was observed in the stigma. The ovules inside the pistil were clearly distinguishable as black dots against the green GFP signal; this indicated that no GFP expression occurred in these cells (Figures 4A-D). A lower level of GFP fluorescence was observed in the stamen and was detectable both in the anther and the filament (Figures 4E,F). The GFP signal in the sepals and petals of the flower was high in the vascular tissues; GFP expression appeared to be significantly greater in the sepal than in the petal (Figures 4G-L).

We also examined the green siliques containing developing seeds. A GFP signal was detected in the valve of the silique and the highest GFP expression was observed at the base of the seeds (Figures 5A-C). A closer look at the seed showed that the high GFP fluorescence appeared to be associated with the seed abscission zone (Figures 5D-F). An intense GFP signal was also found at the base of the siliques, which likely corresponds to the silique abscission zone (Figures 5G-I).

\section{REGULATION OF DES1 EXPRESSION BY EXOGENOUS AUXINS}

The spatial distribution of GFP expression conferred by the DES1 promoter suggests that DES1 is regulated by the hormone auxin (Teale et al., 2006; Wang et al., 2011; Basu et al., 2013; Baylis et al., 2013). Therefore, we analyzed DES1 gene expression in response to the exogenous application of auxins. Seeds were germinated directly on indole-3-acetic acid (IAA) at two different concentrations. After 7 days of growth, the level of DES1 gene expression was determined and compared to the level of gene expression in plants grown in the absence of IAA (Figure 6). We observed a strong and significant reduction in the level of DES1 expression in the presence of the auxin at a lower concentration of $0.1 \mu \mathrm{M}$. In the same samples, we measured the level of OAS-A1 gene expression. OAS-A1 is the cytosolic enzyme that acts in an opposite manner to DES1. A strong and significant induction in the expression level of $O A S-A 1$ was observed (Figure 6).

\section{DISCUSSION}

Recent investigations have changed the view of the A. thaliana L-Cys desulfhydrase 1 (DES1) protein from a minor and auxiliary enzyme belonging to the OASTL protein family to an important and essential enzyme that regulates the homeostasis of cysteine and modulates the generation of sulfide in the 

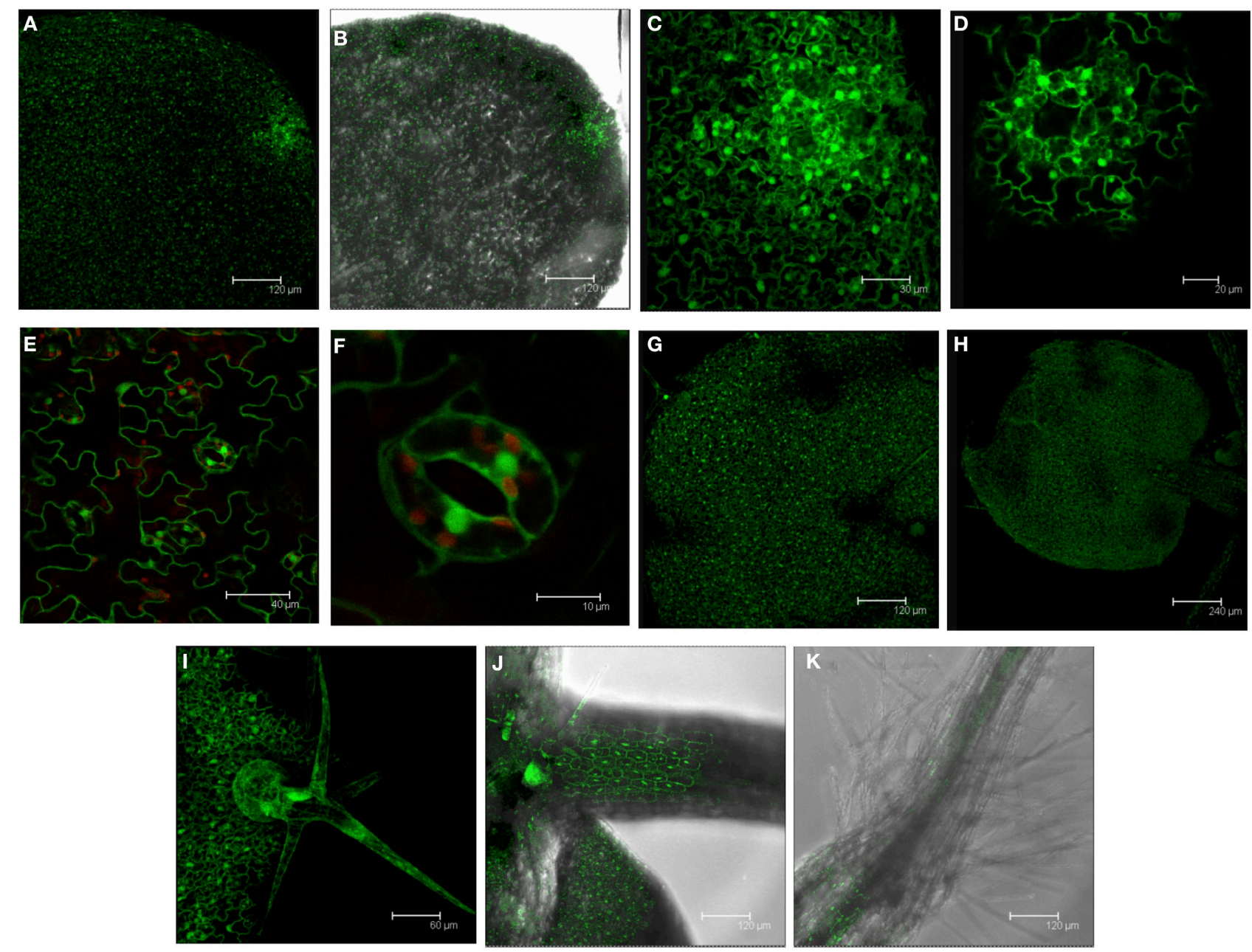

FIGURE 2 | GFP localization in 7-day-old seedlings from the PromDES1-GFP transgenic line. Transgenic Arabidopsis PromDES1-GFP plants were grown on solid MS plates for 7 days. GFP was visualized using confocal fluorescence microscopy. $(\mathbf{A}, \mathbf{B})$ GFP image and the same image with overlapping transmitted light image from the abaxial side of a leaf. (C) Magnification of (A) showing a hydathode pore.

(D) GFP image of a hydathode pore in a different leaf. (E) GFP image with overlapping chloroplast autofluorescence image of epidermal cells on the adaxial side of a leaf. (F) Magnification of (E) showing a stoma. (G,H) GFP images from the adaxial side of two different leaves. (I) GFP image of a trichome. (J) GFP image with overlapping transmitted light image of a petiole. (K) GFP image with overlapping transmitted light image of a root neck. All images shown are Z-stacks of optical sections. cytosol for signaling purposes (Gotor et al., 2014; Romero et al., 2014). Consequently, knowledge of the tissue-specific localization and regulation of the enzyme will help us to understand the mechanisms underlying the specific functions of DES1.

At the protein level, DES1 has very low abundance; this was confirmed by the identificaction of a small number of peptides in proteomic analysis (AtProteome Database). The steady-state DES1 transcript levels are also substantially low. For example, the expression level of the DES1 gene is approximately two orders of magnitude lower that the $O A S-A 1$ expression level; this is illustrated in Figure 6, and easily verifiable using available web resources (www.arabidopsis.org; www.genevestigator. com). When GFP fluorescence is observed using the promoterGFP approach, gene expression driven by the DES1 promoter is relatively high. Fluorescence is mainly observed throughout the whole leaf in early growth stages and in reproductive tissues. These results suggest that DES1 is regulated at posttranscriptional or post-translational level. Such a hypothesis makes sense considering the function of this protein in the generation of sulfide in the cytosol to be used for signaling in important processes such as autophagy (Alvarez et al., 2012a; Gotor et al., 2013; Romero et al., 2013). Sulfide is a toxic molecule; in recent years, it has been further recognized as an important signaling molecule in animal and plant systems (Gadalla and Snyder, 2010; Kimura, 2011; Wang, 2012; Garcia-Mata and Lamattina, 2013; Lisjak et al., 2013; Calderwood and Kopriva, 2014; Hancock and Whiteman, 2014). Therefore, sulfide generation activity in the cytosol should be precisely regulated to avoid deleterious effects. Further investigation will be necessary to determine timing, tissue regulation and the players responsible of this sulfide tuning. Sulfide has been implicated in the regulation of other essential process such as stomatal movement (Garcia-Mata and Lamattina, 

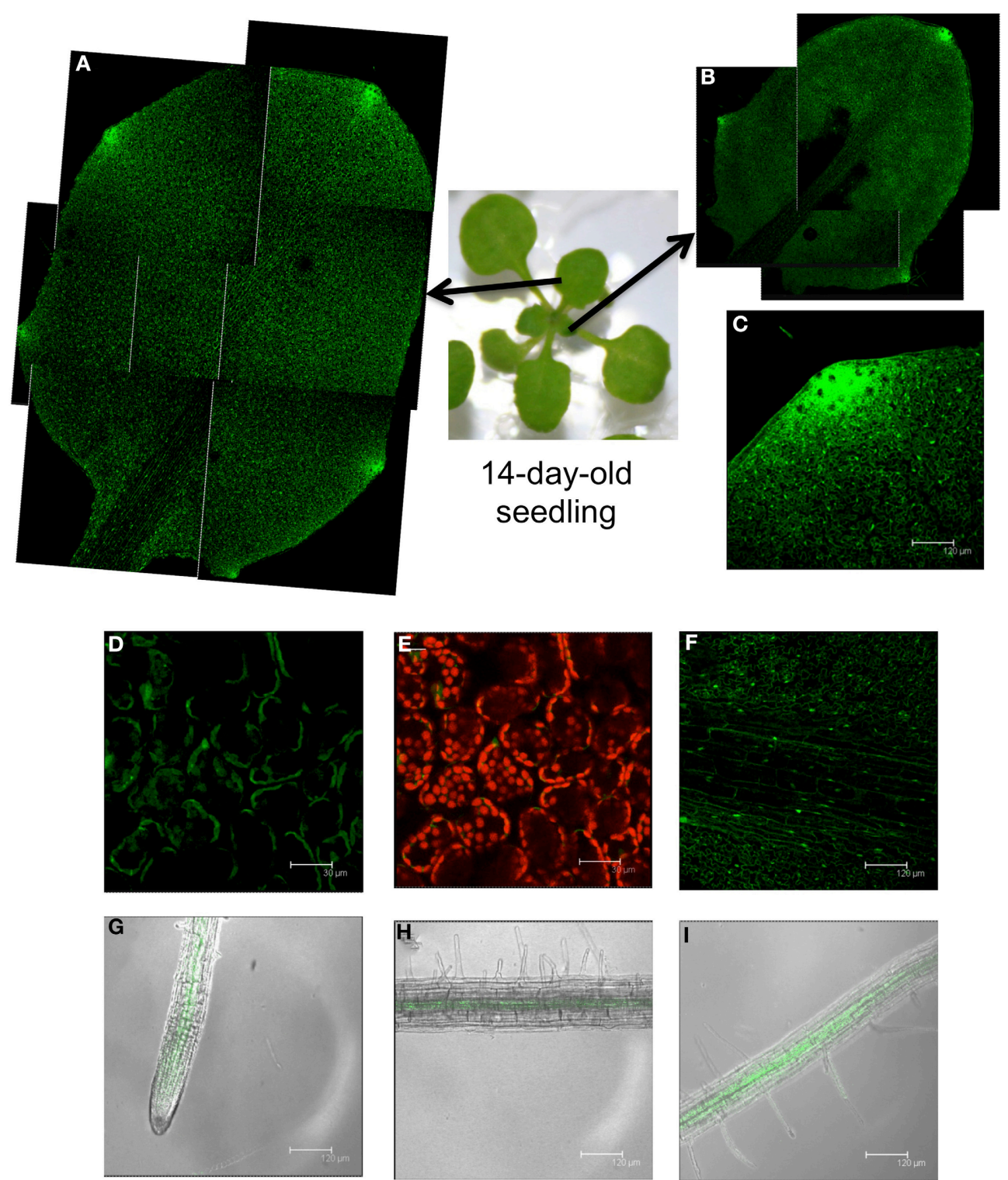

FIGURE 3 | GFP localization in 14-day-old seedlings from the PromDES1-GFP transgenic line. Transgenic Arabidopsis PromDES1-GFP plants were grown on solid MS plates for 14 days. GFP was visualized using confocal fluorescence microscopy. (A,B) Reconstruction of two different leaves from a 14-day-old seedling by joining the GFP images from different sections. (C) GFP image of a hydathode pore. (D,E) GFP image and the same image with overlapping red chloroplast autofluorescence of mesophyll cells. (F) GFP image of a leaf showing the vascular tissue. (G-I) GFP image with overlapping transmitted light image from different root sections. All images shown are Z-stacks of optical sections.
2010; Lisjak et al., 2010). Very recently, the involvement of DES1 in the ABA-dependent signaling network in guard cells and the requirement for DES1 in $\mathrm{ABA}$-dependent $\mathrm{NO}$ production have been demonstrated (Scuffi et al., 2014). These data suggest that the DES1 protein may be hormonally regulated and may crosstalk with other signaling molecules.

The present study demonstrates that the maximum DES1 expression occurs at the initial (seedling) and final (maturity) stages of plant development. This suggests a specific role for DES1 at these developmental stages. These data fit well with data gathered using des 1 null mutants, in which phenotypic differences were observed at these stages. ROS production was practically unchanged after cadmium treatment in des1 mutant seedlings, in contrast with wild type seedlings. Consequently, the DES1 mutation produces an enhanced tolerance to cadmium and $\mathrm{H}_{2} \mathrm{O}_{2}$ stress conditions (Alvarez et al., 2010). At maturity, mutation in the DES1 gene leads to premature leaf senescence and promotes the accumulation and lipidation of the ATG8 protein; ATG8 is typically associated with the induction of autophagy. The transcriptional profile of the des1 mutant corresponds with the observed premature senescence and induced autophagy phenotypes. Most important, the DES1 mutation significantly alters the 

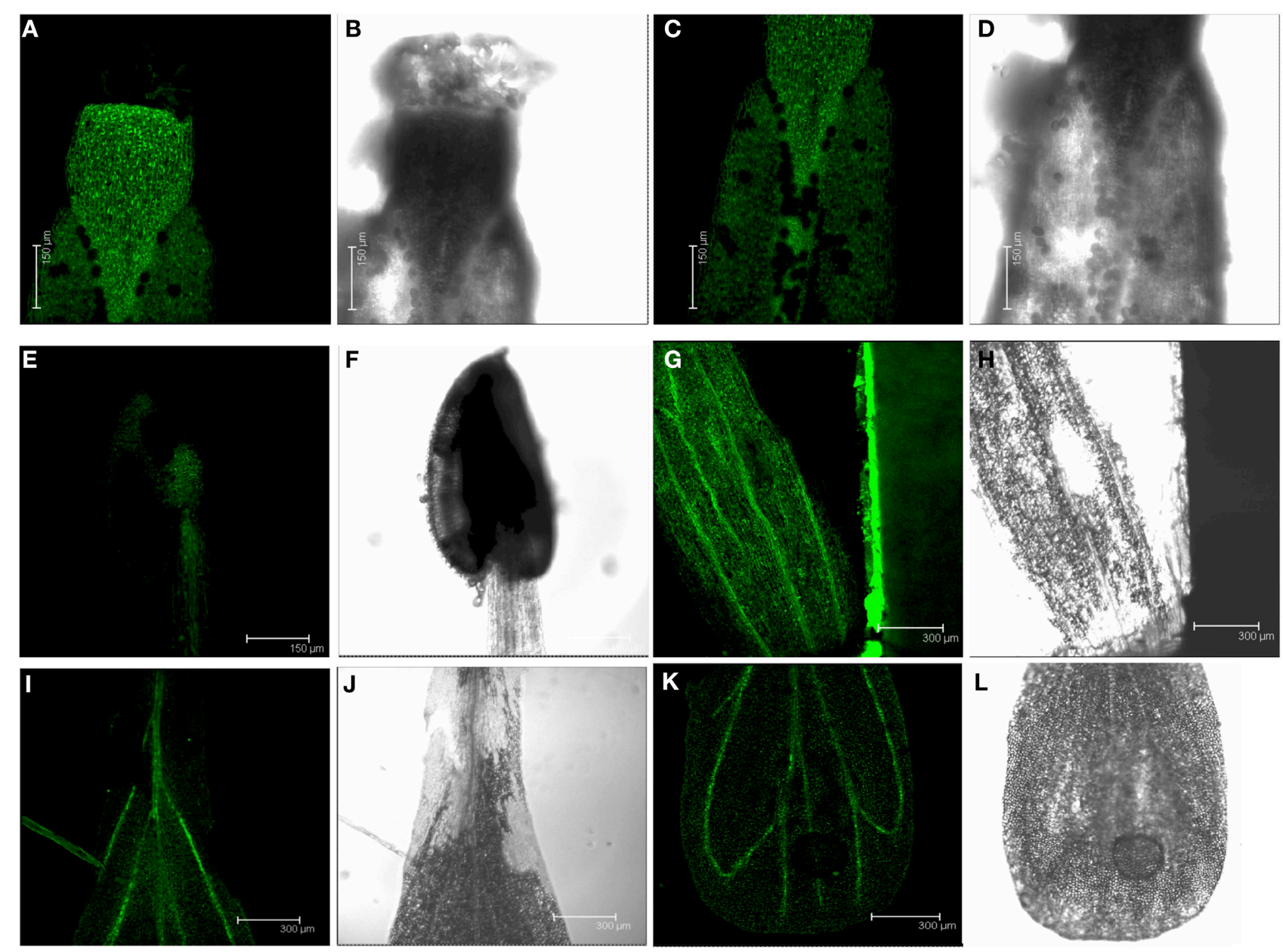

FIGURE 4 | GFP localization in the reproductive tissues of plants from the PromDES1-GFP transgenic line. Transgenic Arabidopsis PromDES1-GFP plants were grown in soil under physiological growth conditions for 35-40 days. GFP was visualized using confocal fluorescence microscopy. (A,B) GFP and transmitted light images of the

upper part of a pistil. (C,D) GFP and transmitted light images of the base of a pistil. (E,F) GFP and transmitted light images of a stamen. $(\mathbf{G}, \mathbf{H})$ GFP and transmitted light images of a sepal. (I-L) GFP and transmitted light images of whole petal. All images shown are Z-stacks of optical sections.

transcriptional profile at the late growth stage. When transcriptomic analysis was performed using leaves from plants grown for $20 \mathrm{~d}$ (growth stage 3.9), only 16 genes in the des 1 mutant were differentially expressed compared to wild type plants. In contrast, the des 1 transcriptional profile changed dramatically compared to wild type in leaves from plants grown for $30 \mathrm{~d}$ (growth stage 6.3). The normalized data revealed that 1614 genes were differentially expressed in the mutant compared to the wild type (Alvarez et al., 2012a). Consequently, the function of DES1 seems to be critical at this late growth stage.

An examination of GFP expression driven by the DES1 promoter in vegetative tissues reveals that the highest GFP signal occurs in the hydathode pores distributed along the margin of the leaf; the number of pores increases with the leaf size. Hydathodes are specialized pore-like structures that act as the exit point in vascular tissues. At these sites, water and ions are released from the xylem. It has also suggested that hydathodes are involved in ion reabsorption to other tissues through the phloem (Nagai et al.,
2013). In addition, the hydathodes are open pores similar to stomata (Nagai et al., 2013). The Arabidopsis basic helix-loop-helix (bHLH) protein MUTE, which is a master regulator of stomatal differentiation, is also required for the production of hydathodes (Pillitteri et al., 2008). We have detected a significant GFP fluorescence signal localized to the cytoplasm of guard cells, which suggests the DES1 protein or the sulfide generated by DES1 has a specific function in these pore structures in Arabidopsis leaves. This suggestion is reinforced by our recent findings that show DES1 is required for ABA-dependent stomatal closure and the sulfide generated by DES1 acts upstream of nitric oxide in this signaling network (Scuffi et al., 2014).

The DES1 promoter also confers strong GFP expression inside the trichomes; this result supports numerous reports that have demonstrated the significance of this cell type in relation to sulfur metabolism. In situ hybridization studies in combination with determinations of glutathione content by confocal microscopy demonstrated that highly active glutathione biosynthesis occurs 

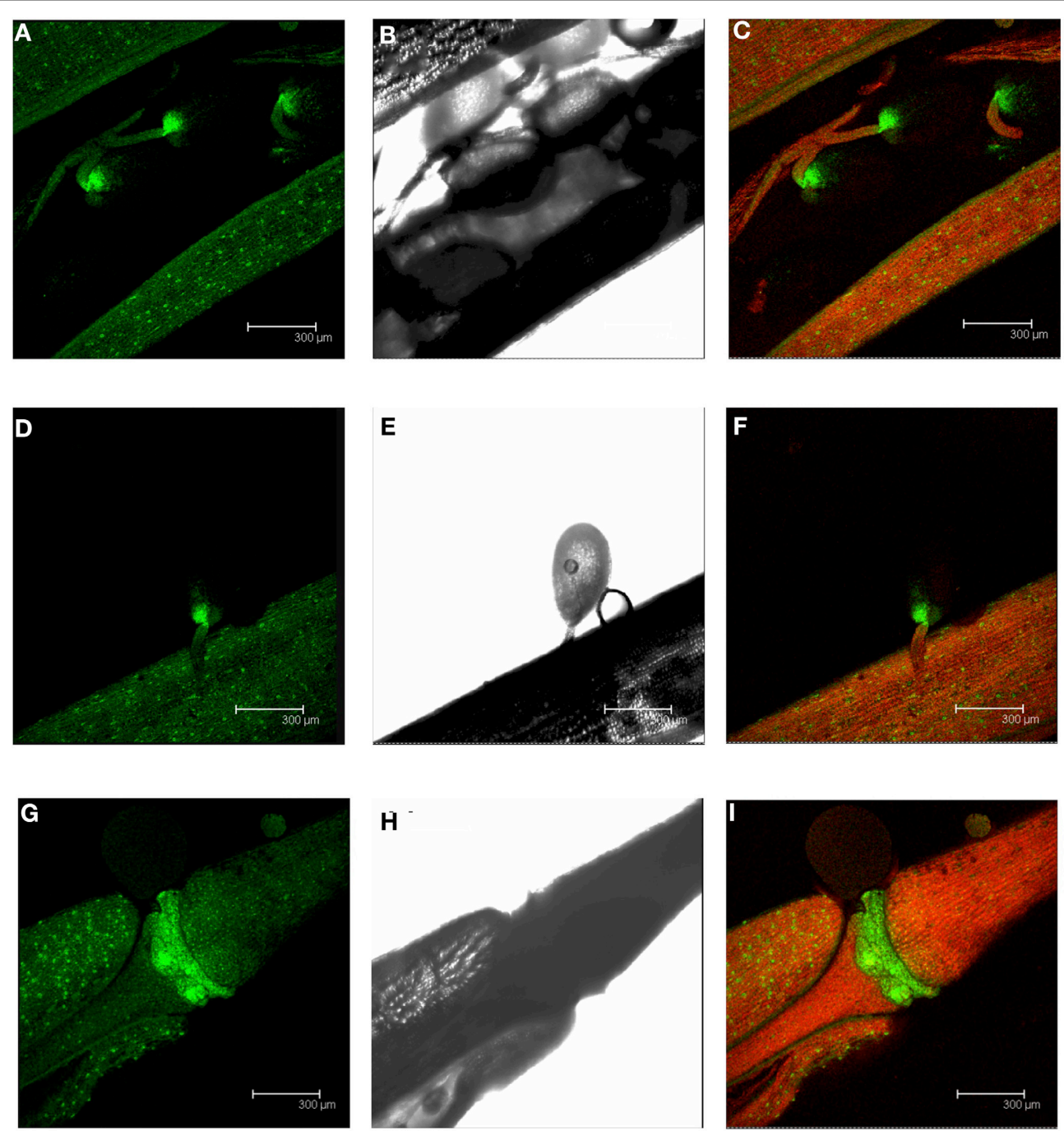

FIGURE 5 | GFP localization in developing siliques of plants from the PromDES1-GFP transgenic line. Transgenic Arabidopsis PromDES1-GFP plants were grown in soil under physiological growth conditions for 40 days. GFP was visualized using confocal fluorescence microscopy. (A-C) GFP image, transmitted light image, the same GFP image with overlapping transmitted light and red chloroplast autofluorescence image of a developing silique containing several immature seeds. (D-F) GFP image, transmitted light image, the same GFP image with overlapping transmitted light and red chloroplast autofluorescence image of an immature seed. (G-I) GFP image, transmitted light image, the same GFP image with overlapping transmitted light and red chloroplast autofluorescence image in the abscission zone of a silique. All images shown are Z-stacks of optical sections. in Arabidopsis trichome cells (Gotor et al., 1997; Gutierrez-Alcala et al., 2000). Furthermore, protein profiling performed in this specific cell type also identified an important number of proteins involved in sulfur metabolism (Wienkoop et al., 2004). This trichome-specific expression driven by the DES1 promoter is similar to expression driven by the OAS-A1 promoter (GutierrezAlcala et al., 2005). These findings suggest that, in this cell type, the homeostasis of cysteine is important and is modulated by the enzymes OAS-A1 and DES1; OAS-A1 catalyzes the synthesis of cysteine and DES1 catalyzes the degradation of cysteine.

In this work, a detailed analysis of the expression of a reporter gene driven by the promoter of a gene encoding an enzyme involved in plant sulfur assimilation was performed for the first time. The localization of reporter expression conferred by such promoters in reproductive tissues was previously unknown. In open flowers, the DES1 promoter confers high GFP expression, which occurs mainly in the pistil, sepal, and petal. Weaker GFP signals were observed in the stamen. The presence of OAS-A1 transcripts was also detected in flowers by in situ hybridizations; this finding was analogous to our observations in trichomes (Gotor et al., 1997).

When siliques were analyzed, strong GFP fluorescence was detected in the presumed abscission zones at the bases of the siliques and seeds. Cell separation is a process highly regulated by plant hormones. Ethylene, JA, and ABA act together to regulate organ abscission (Ogawa et al., 2009). Auxin is also involved in many abscission events (Basu et al., 2013). 


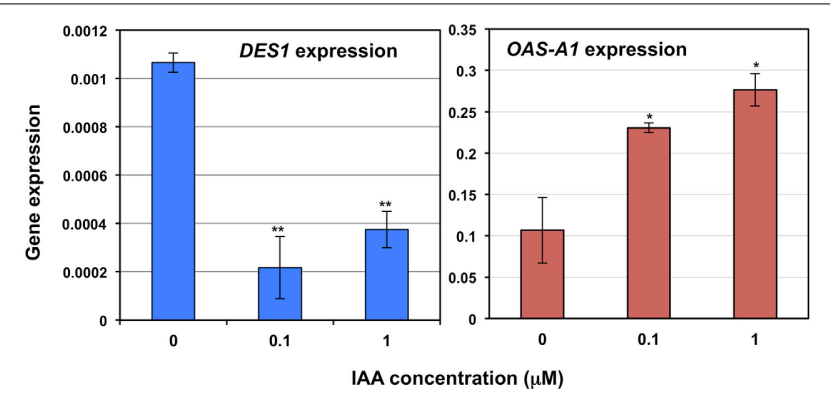

FIGURE 6 | The effect of exogenous auxins on the expression levels of DES1 and OAS-A1 genes in wild type plants. Wild type Arabidopsis plants were grown on solid MS plates for 7 days in either the absence or presence of IAA at the indicated concentrations. Whole seedlings were then collected for real-time RT-PCR analysis. The DES1 and OAS-A1

transcript levels were normalized to the internal control, the constitutively expressed UBQ10 gene. Data shown are mean values \pm SD from three independent analyses. The one-factor analysis of variance (ANOVA) statistical analysis of the data was performed using the program OriginPro 7.5. ${ }^{* *} P<0.01 ; * P<0.05$.

In general, the tissue-specific expression pattern of GFP conferred by the DES1 promoter supports the hormonal regulation of DES1. We have identified cis-elements located within the promoter sequence that correlate with specific hormonal regulation; these include ABA response elements (DPBF binding site motifs), drought response elements (DRE-like promoter motifs), and auxin response elements (ARF1 binding site motifs), and several stress-responsive binding site motifs. The DES1 promoter also contains a number of light responsive elements, regulatory elements involved in flowering, such as the LFY consensus binding site motif, and numerous RAV1-A binding site motifs involved in leaf maturation and senescence. The presence of these motifs in the DES1 promoter corroborates the transcriptomic data from des1 null mutants, which suggest DES1 plays an important role in mature plants.

The guard cell-specific expression of GFP suggests that DES1 is regulated by $\mathrm{ABA}$, and this finding has been recently demonstrated when the stomatal closure in des1 null mutants was analyzed (Scuffi et al., 2014). Wild type plants closed the stomata in response to exogenous $\mathrm{ABA}$, and des 1 mutants were unable to close the stomata. This lack of response to ABA in des1 mutants was restored by genetic complementation or by the exogenous application of sulfide. Taken together, these data indicate that DES1 is required for ABA-dependent stomatal closure. It has been demonstrated that DES1 is regulated by ABA at the transcriptional level, specifically in guard cells (Scuffi et al., 2014). Interestingly, it was also observed that the OAS-A1 regulation in response to salt stress is mediated by $\mathrm{ABA}$ (Barroso et al., 1999).

GFP expression driven by the DES1 promoter localizes to sites of high auxin concentration, such as hydathodes (Teale et al., 2006; Wang et al., 2011). This suggests that DES1 may be regulated by auxin, however, we do not observe any root phenotype in the des 1 mutants. At the transcriptional level, we have observed clear repression in the DES1 transcript level in response to the exogenous application of auxins; the opposite behavior was observed with the $O A S$ - $A 1$ transcript. The GFP accumulation pattern seems contradictory with the down regulation of DES1 gene expression by auxin. However, the mechanisms controlling auxin action are very complex and involve auxin biosynthesis, conjugation, catabolism, and transport. At present, we are unable to decipher the specific aspect where DES1 is involved, although we suggest a crosstalk between DES1 and the auxin-signaling pathway.

Auxin regulates a variety of physiological and developmental processes in plants, including senescence. However, different lines of evidence suggest that auxin delays senescence (Lim et al., 2010; Kim et al., 2011) and other evidence suggest that auxin promotes senescence (Hou et al., 2013). Regardless, the premature leaf senescence phenotype observed in the des 1 mutants and also the transcriptional profile that shows the altered expression of auxin-responsive and small auxin upregulated (SAUR) genes suggest that DES1 is regulated by auxin (Gene Expression Omnibus repository GSE32566) (Alvarez et al., 2012a).

The regulatory relationship between sulfur signaling and auxins was previously demonstrated by analyzing the transcriptional responses of plants to sulfur deficiency. The genes involved in the auxin biosynthesis pathway are up-regulated under sulfur deficiency; this suggests that auxin is involved in the sulfur starvation response (Hirai et al., 2003; Nikiforova et al., 2003). Different sulfur starvation response factors related to auxin signaling have been analyzed, and it was concluded that auxin-related transcriptional regulators coordinate the metabolic shifts induced by sulfur starvation (Falkenberg et al., 2008).

\section{ACKNOWLEDGMENTS}

This work was funded in part by the European Regional Development Fund through the Ministerio de Economia $y$ Competitividad (grant no. BIO2013-44648-P) and the Junta de Andalucía (grant no. CVI-7190). Ana M. Laureano-Marín thanks the Ministerio de Economia y Competitividad for fellowship support through the Formación de Personal Investigador program. We thank Dr. Alicia Orea for confocal microscopy service.

\section{SUPPLEMENTARY MATERIAL}

The Supplementary Material for this article can be found online at: http://www.frontiersin.org/journal/10.3389/fpls.2014.00683/ abstract

\section{REFERENCES}

Alvarez, C., Calo, L., Romero, L. C., Garcia, I., and Gotor, C. (2010). An Oacetylserine(thiol)lyase homolog with L-cysteine desulfhydrase activity regulates cysteine homeostasis in Arabidopsis. Plant Physiol. 152, 656-669. doi: 10.1104/pp.109.147975

Alvarez, C., Garcia, I., Moreno, I., Perez-Perez, M. E., Crespo, J. L., Romero, L. C., et al. (2012a). Cysteine-generated sulfide in the cytosol negatively regulates autophagy and modulates the transcriptional profile in Arabidopsis. Plant Cell 24, 4621-4634. doi: 10.1105/tpc.112.105403

Alvarez, C., Garcia, I., Romero, L. C., and Gotor, C. (2012b). Mitochondrial sulfide detoxification requires a functional isoform $\mathrm{O}$-acetylserine(thiol)lyase $\mathrm{C}$ in Arabidopsis thaliana. Mol. Plant 5, 1217-1226. doi: 10.1093/mp/sss043

Barroso, C., Romero, L. C., Cejudo, F. J., Vega, J. M., and Gotor, C. (1999). Salt-specific regulation of the cytosolic O-acetylserine(thiol)lyase gene from Arabidopsis thaliana is dependent on abscisic acid. Plant Mol. Biol. 40, 729-736. doi: 10.1023/A:1006285016296 
Basu, M. M., Gonzalez-Carranza, Z. H., Azam-Ali, S., Tang, S., Shahid, A. A., and Roberts, J. A. (2013). The manipulation of auxin in the abscission zone cells of Arabidopsis flowers reveals that indoleacetic acid signaling is a prerequisite for organ shedding. Plant Physiol. 162, 96-106. doi: 10.1104/pp.113. 216234

Baylis, T., Cierlik, I., Sundberg, E., and Mattsson, J. (2013). SHORT INTERNODES/STYLISH genes, regulators of auxin biosynthesis, are involved in leaf vein development in Arabidopsis thaliana. New Phytol. 197, 737-750. doi: 10.1111/nph.12084

Boyes, D. C., Zayed, A. M., Ascenzi, R., McCaskill, A. J., Hoffman, N. E., Davis, K. R., et al. (2001). Growth stage-based phenotypic analysis of Arabidopsis: a model for high throughput functional genomics in plants. Plant Cell 13, 1499-1510. doi: $10.1105 /$ tpc.13.7.1499

Calderwood, A., and Kopriva, S. (2014). Hydrogen sulfide in plants: from dissipation of excess sulfur to signaling molecule. Nitric Oxide. 41, 72-78. doi: 10.1016/j.niox.2014.02.005

Clough, S. J., and Bent, A. F. (1998). Floral dip: a simplified method for Agrobacterium-mediated transformation of Arabidopsis thaliana. Plant J. 16, 735-743. doi: 10.1046/j.1365-313x.1998.00343.x

Curtis, M. D., and Grossniklaus, U. (2003). A gateway cloning vector set for highthroughput functional analysis of genes in planta. Plant Physiol. 133, 462-469. doi: 10.1104/pp.103.027979

Falkenberg, B., Witt, I., Zanor, M. I., Steinhauser, D., Mueller-Roeber, B., Hesse, H., et al. (2008). Transcription factors relevant to auxin signalling coordinate broad-spectrum metabolic shifts including sulphur metabolism. J. Exp. Bot. 59, 2831-2846. doi: 10.1093/jxb/ern144

Gadalla, M. M., and Snyder, S. H. (2010). Hydrogen sulfide as a gasotransmitter. J. Neurochem. 113, 14-26. doi: 10.1111/j.1471-4159.2010. 06580.x

Garcia, I., Castellano, J. M., Vioque, B., Solano, R., Gotor, C., and Romero, L. C. (2010). Mitochondrial \{beta\}-cyanoalanine synthase is essential for root hair formation in Arabidopsis thaliana. Plant Cell 22, 3268-3279. doi: 10.1105/tpc. 110.076828

Garcia-Mata, C., and Lamattina, L. (2010). Hydrogen sulphide, a novel gasotransmitter involved in guard cell signalling. New Phytol. 188, 977-984. doi: 10.1111/j.1469-8137.2010.03465.x

Garcia-Mata, C., and Lamattina, L. (2013). Gasotransmitters are emerging as new guard cell signaling molecules and regulators of leaf gas exchange. Plant Sci. 201-202, 66-73. doi: 10.1016/j.plantsci.2012.11.007

Gotor, C., Cejudo, F. J., Barroso, C., and Vega, J. M. (1997). Tissue-specific expression of ATCYS-3A, a gene encoding the cytosolic isoform of Oacetylserine(thiol)lyase in Arabidopsis. Plant J. 11, 347-352. doi: 10.1046/j.1365313X.1997.11020347.x

Gotor, C., Garcia, I., Crespo, J. L., and Romero, L. C. (2013). Sulfide as a signaling molecule in autophagy. Autophagy 9, 609-611. doi: 10.4161/auto. 23460

Gotor, C., Laureano-Marin, A. M., Moreno, I., Aroca, A., García, I., and Romero, L. C. (2014). Signaling in the plant cytosol: cysteine or sulfide? Amino Acids. doi: 10.1007/s00726-014-1786-z. [Epub ahead of print]

Gutierrez-Alcala, G., Calo, L., Gros, F., Caissard, J. C., Gotor, C., and Romero, L. C. (2005). A versatile promoter for the expression of proteins in glandular and non-glandular trichomes from a variety of plants. J. Exp. Bot. 56, 2487-2494. doi: $10.1093 /$ jxb/eri241

Gutierrez-Alcala, G., Gotor, C., Meyer, A. J., Fricker, M., Vega, J. M., and Romero, L. C. (2000). Glutathione biosynthesis in Arabidopsis trichome cells. Proc. Natl. Acad. Sci. U.S.A. 97, 11108-11113. doi: 10.1073/pnas.190334497

Hancock, J. T., and Whiteman, M. (2014). Hydrogen sulfide and cell signaling: team player or referee? Plant Physiol. Biochem. 78, 37-42. doi: 10.1016/j.plaphy.2014.02.012

Hirai, M. Y., Fujiwara, T., Awazuhara, M., Kimura, T., Noji, M., and Saito, K. (2003) Global expression profiling of sulfur-starved Arabidopsis by DNA macroarray reveals the role of O-acetyl-1-serine as a general regulator of gene expression in response to sulfur nutrition. Plant J. 33, 651-663. doi: 10.1046/j.1365313X.2003.01658.x

Hou, K., Wu, W., and Gan, S. S. (2013). SAUR36, a small auxin up RNA gene, is involved in the promotion of leaf senescence in Arabidopsis. Plant Physiol. 161, 1002-1009. doi: 10.1104/pp.112.212787

Kabil, O., and Banerjee, R. (2010). Redox biochemistry of hydrogen sulfide. J. Biol. Chem. 285, 21903-21907. doi: 10.1074/jbc.R110.128363
Kim, J. I., Murphy, A. S., Baek, D., Lee, S. W., Yun, D. J., Bressan, R. A., et al. (2011). YUCCA6 over-expression demonstrates auxin function in delaying leaf senescence in Arabidopsis thaliana. J. Exp. Bot. 62, 3981-3992. doi: 10.1093/jxb/ err094

Kimura, H. (2011). Hydrogen sulfide: its production and functions. Exp. Physiol. 96, 833-835. doi: 10.1113/expphysiol.2011.057455

Krueger, S., Niehl, A., Lopez Martin, M. C., Steinhauser, D., Donath, A., Hildebrandt, T., et al. (2009). Analysis of cytosolic and plastidic serine acetyltransferase mutants and subcellular metabolite distributions suggests interplay of the cellular compartments for cysteine biosynthesis in Arabidopsis. Plant Cell Environ. 32, 349-367. doi: 10.1111/j.1365-3040.2009. 01928.x

Lim, P. O., Lee, I. C., Kim, J., Kim, H. J., Ryu, J. S., Woo, H. R., et al. (2010). Auxin response factor 2 (ARF2) plays a major role in regulating auxin-mediated leaf longevity. J. Exp. Bot. 61, 1419-1430. doi: 10.1093/jxb/ erq010

Lisjak, M., Srivastava, N., Teklic, T., Civale, L., Lewandowski, K., Wilson, I., et al. (2010). A novel hydrogen sulfide donor causes stomatal opening and reduces nitric oxide accumulation. Plant Physiol. Biochem. 48, 931-935. doi: 10.1016/j.plaphy.2010.09.016

Lisjak, M., Teklic, T., Wilson, I. D., Whiteman, M., and Hancock, J. T. (2013). Hydrogen sulfide: environmental factor or signalling molecule? Plant Cell Environ. 36, 1607-1616. doi: 10.1111/pce.12073

Nagai, M., Ohnishi, M., Uehara, T., Yamagami, M., Miura, E., Kamakura, M., et al. (2013). Ion gradients in xylem exudate and guttation fluid related to tissue ion levels along primary leaves of barley. Plant Cell Environ. 36, 1826-1837. doi: $10.1111 /$ pce. 12090

Nikiforova, V., Freitag, J., Kempa, S., Adamik, M., Hesse, H., and Hoefgen, R. (2003). Transcriptome analysis of sulfur depletion in Arabidopsis thaliana: interlacing of biosynthetic pathways provides response specificity. Plant J. 33, 633-650. doi: 10.1046/j.1365-313X.2003.01657.x

Ogawa, M., Kay, P., Wilson, S., and Swain, S. M. (2009). ARABIDOPSIS DEHISCENCE ZONE POLYGALACTURONASE1 (ADPG1), ADPG2, and QUARTET2 are polygalacturonases required for cell separation during reproductive development in Arabidopsis. Plant Cell 21, 216-233. doi: 10.1105/tpc.108.063768

Pillitteri, L. J., Bogenschutz, N. L., and Torii, K. U. (2008). The bHLH protein, MUTE, controls differentiation of stomata and the hydathode pore in Arabidopsis. Plant Cell Physiol. 49, 934-943. doi: 10.1093/pcp/ pcn067

Riemenschneider, A., Wegele, R., Schmidt, A., and Papenbrock, J. (2005). Isolation and characterization of a D-cysteine desulfhydrase protein from Arabidopsis thaliana. FEBS J. 272, 1291-1304. doi: 10.1111/j.1742-4658.2005. 04567.x

Romero, L. C., Aroca, M. A., Laureano-Marin, A. M., Moreno, I., Garcia, I., and Gotor, C. (2014). Cysteine and cysteine-related signaling pathways in Arabidopsis thaliana. Mol. Plant 7, 264-276. doi: 10.1093/mp/ sst168

Romero, L. C., Garcia, I., and Gotor, C. (2013). L-Cysteine Desulfhydrase 1 modulates the generation of the signaling molecule sulfide in plant cytosol. Plant Signal. Behav. 8:e24007. doi: 10.4161/psb.24007

Scuffi, D., Álvarez, C. Laspina, N., Gotor, C., Lamattina, L., and Garcia-Mata, C. (2014). Hydrogen sulfide generated by L-cysteine desulfhydrase acts upstream of nitric oxide to modulate ABA-dependent stomatal closure. Plant Physiol. 166 , doi: 10.1104/pp.114.245373. [Epub ahead of print]

Seibel, N. M., Eljouni, J., Nalaskowski, M. M., and Hampe, W. (2007) Nuclear localization of enhanced green fluorescent protein homomultimers. Anal. Biochem. 368, 95-99. doi: 10.1016/j.ab.2007. 05.025

Takahashi, H., Kopriva, S., Giordano, M., Saito, K., and Hell, R. (2011). Sulfur assimilation in photosynthetic organisms: molecular functions and regulations of transporters and assimilatory enzymes. Annu. Rev. Plant Biol. 62, 157-184. doi: 10.1146/annurev-arplant-042110-103921

Teale, W. D., Paponov, I. A., and Palme, K. (2006). Auxin in action: signalling, transport and the control of plant growth and development. Nat. Rev. Mol. Cell Biol. 7, 847-859. doi: 10.1038/nrm2020

Wang, R. (2012). Physiological implications of hydrogen sulfide: a whiff exploration that blossomed. Physiol. Rev. 92, 791-896. doi: 10.1152/physrev. 00017.2011 
Wang, W., Xu, B., Wang, H., Li, J., Huang, H., and Xu, L. (2011). YUCCA genes are expressed in response to leaf adaxial-abaxial juxtaposition and are required for leaf margin development. Plant Physiol. 157, 1805-1819. doi: 10.1104/pp.111.186395

Wienkoop, S., Zoeller, D., Ebert, B., Simon-Rosin, U., Fisahn, J., Glinski, M., et al. (2004). Cell-specific protein profiling in Arabidopsis thaliana trichomes: identification of trichome-located proteins involved in sulfur metabolism and detoxification. Phytochemistry 65, 1641-1649. doi: 10.1016/j.phytochem.2004.03.026

Conflict of Interest Statement: The authors declare that the research was conducted in the absence of any commercial or financial relationships that could be construed as a potential conflict of interest.
Received: 29 October 2014; accepted: 16 November 2014; published online: 04 December 2014.

Citation: Laureano-Marín AM, García I, Romero LC and Gotor C (2014) Assessing the transcriptional regulation of L-cysteine desulfhydrase 1 in Arabidopsis thaliana. Front. Plant Sci. 5:683. doi: 10.3389/fpls.2014.00683

This article was submitted to Plant Physiology, a section of the journal Frontiers in Plant Science.

Copyright (C) 2014 Laureano-Marin, García, Romero and Gotor. This is an openaccess article distributed under the terms of the Creative Commons Attribution License (CC BY). The use, distribution or reproduction in other forums is permitted, provided the original author(s) or licensor are credited and that the original publication in this journal is cited, in accordance with accepted academic practice. No use, distribution or reproduction is permitted which does not comply with these terms. 